\title{
On feedback queueing system with reneging and retention of reneged customers, multiple working vacations and Bernoulli schedule vacation interruption
}

Received: 12 March 2016 / Accepted: 28 November 2016 / Published online: 20 January 2017

(C) The Author(s) 2017. This article is published with open access at Springerlink.com

\begin{abstract}
This paper presents an analysis of a Markovian feedback queueing system with reneging and retention of reneged customers, multiple working vacations and Bernoulli schedule vacation interruption, where customers' impatience is due to the servers' vacation. The reneging times are assumed to be exponentially distributed. After the completion of service, each customer may reenter the system as a feedback customer for receiving another regular service with some probability or leave the system. A reneged customer can be retained in many cases by employing certain convincing mechanisms to stay in queue for completion of service. Thus, a reneged customer can be retained in the queueing system with some probability or he may leave the queue without receiving service. We establish the stationary analysis of the system. The probability generating functions of the stationary state probabilities is obtained, we deduce the explicit expressions of the system sizes when the server is in a normal service period and in a Bernoulli schedule vacation interruption, respectively. Various performance measures of the system are derived. Finally, we present some numerical examples to demonstrate how the various parameters of the model influence the behavior of the system.
\end{abstract}

\section{Mathematics Subject Classification $\quad 60 \mathrm{~K} 25 \cdot 68 \mathrm{M} 20 \cdot 90 \mathrm{~B} 22$}

A. A. Bouchentouf $(\varangle)$

Department of Mathematics, Mathematics Laboratory, Djillali Liabes University of Sidi Bel Abbes, B. P. 89, 22000 Sidi Bel Abbes, Algeria

E-mail: bouchentouf_amina@yahoo.fr

L. Yahiaoui

Department of Mathematics, Dr. Moulay Taher University, 20000 Saida, Algeria 


\section{Introduction}

Queueing is a prevalent phenomenon in our daily lives. At this time, queueing theory is very important in studying scheduling and system performance, it is also an all powerful tool to solve various problems in many complex systems, such as computer systems, telecommunication systems, call centers, flexible manufacturing systems and service systems. During the past few decades, there has been increasing interest in studying queueing systems.

Queueing systems with customers' impatience and server vacations have been widely studied because of their wide applications in real-life congestion problems such as communication systems, telecommunication systems, traffic systems, and manufacturing/production systems.

The customer's impatient behavior should be needed in the study of queueing system to model real conditions precisely. Occasional operation of a service may be economically invoking when entire time service would result in substantial server idle time or would prevent the utilization of the server in different productive capacities. On the other hand, the server remaining not working for periods of time might gain the probability of customer losses due to balking and reneging Goswami [10].

Vacation queues have been greatly analyzed, Ke et al. [13] provided a succinct summary of the most recent research works on vacation queueing systems in the last decade, Tian and Zhang [25] discussed many variations of vacation policy, and a variety of typical vacation model applications that include call centers with multi-task employees, customized manufacturing, telecommunication systems, maintenance activities, etc are also studied. Yue et al. [27] presented an analysis for an $M / M / 1 / N$ queueing system with balking, reneging and server vacations. By using the Markov process method, authors developed the equations of the steady state probabilities, then, they derived the matrix form solution of the steady-state probabilities, and gave some performance measures of the system, after that they formulated a cost model to determine the optimal service rate.

There are several situations where the server stays active during the vacation period. The server can provide service at a lower speed during the working vacation period instead of stopping service completely. If the queue is empty at the end of a vacation, the server takes another vacation; otherwise a service period begins with normal service rate Goswami [10].

Queueing systems with vacation interruption have been investigated by many authors, Baba [6] studied an $M / P H / 1$ queue with phase type working vacation and vacation interruption where the vacation time follows a phase type distribution, Chen et al. [7] Considered a $G I / M / 1$ queue with phase-type working vacations and vacation interruption where the vacation time follows a phase-type distribution. Li and Tian [18] studied the $M / M / 1$ queue with working vacations and vacation interruptions, Zhang and Hou [29] analyzed an $M / G / 1$ queue with a working vacations and vacation interruption. Using the method of a supplementary variable and the matrix-analytic method, authors obtained the queue length distribution and service status at an arbitrary epoch under steady state conditions. Zhang and Shi [30] presented an $M / M / 1$ queue with Bernoulli schedule vacation and vacation interruption. Altman and Yechiali [2] considered the impatience of customers only when the servers are on vacation and unavailable for service. Selvaraju and Goswami [21] analyzed impatient customers in a single server Markovian queue with single and multiple working vacations.

Many practical queueing systems especially those with balking and reneging have been widely applied to many real-life problems, such as the situations involving impatient telephone switchboard customers, the hospital emergency rooms handling critical patients, and the inventory systems with storage of perishable goods Robert [19]. Haight [11] considered an $M / M / 1$ queue with balking. An $M / M / 1$ queue with customers reneging was also proposed by Haight [12]. The combined effects of balking and reneging in an $M / M / 1 / N$ queue have been investigated by Ancker and Gafarian [3,4]. Abou-EI-Ata and Hariri [1] considered the multiple servers queueing system $M / M / c / N$ with balking and reneging. Wang and Chang [26] extended this work to study an $M / M / c / N$ queue with balking, reneging and server breakdowns. Laxmi et al. [17] studied $M / M / 1 / N$ working vacations queue with balking and reneging. Yue et al. [28] analyzed an $M / M / 1$ queueing system with working vacations and impatient customers, authors derived the probability generating functions of the number of customers in the system when the server is in a service period and a working vacation, respectively, then they obtained the closed-form expressions for various performance measures.

Feedback in queueing literature represents customer dissatisfaction because of inappropriate quality of service. In case of feedback, after getting partial or incomplete service, customer retries for service. In computer communication, the transmission of protocol data unit is sometimes repeated due to occurrence of an error. This usually happens because of non-satisfactory quality of service. Rework in industrial operations is also an example of a queue with feedback Kumar and Sharma [16]. Takacs [23] studied queue with feedback to 
determine the stationary process for the queue size and the first two moments of the distribution function of the total time spent in the system by a customer. In [8] D'Avignon and Disney studied single server queues with state dependent feedback. Santhakumaran and Thangaraj [20] considered a single server feedback queue with impatient and feedback customers, they studied $M / M / 1$ queueing model for queue length at arrival epochs and obtained result for stationary distribution, mean and variance of queue length. Thangaraj and Vanitha [24] obtained transient solution of $M / M / 1$ feedback queue with catastrophes using continued fractions, the steady-state solution, moments under steady state and busy period analysis were calculated. Ayyapan et al. [5] studied $M / M / 1$ retrial queueing system with loss and feedback under non preemptive priority service by matrix geometric method. Kumar and Sharma [14] studied a single server queueing system with retention of reneged customers. Kumar and Sharma [15] studied a single server queueing system with retention of reneged customers and balking. Sharma and Kumar [22] considered a single server, finite capacity Markovian feedback queue with reneging, balking and retention of reneged customers in which the inter-arrival and service times follow exponential distribution. Mahdy El-Paoumy and Hossam Nabwey [9] studied the $M / M / 2 / N$ queue with general balk function, reneging and two heterogeneous servers. In [10], Goswami analyzed customers' impatience in Markovian queueing system with multiple working vacations and Bernoulli schedule vacation interruption, where customers' impatience is due to the servers' vacation.

In this paper, we consider a single-server Markovian feedback queueing system with reneging and retention of reneged customers, multiple working vacations and Bernoulli schedule vacation interruption, where customers' impatience is due to the servers' vacation. During the working vacation period, if there are customers in the queue, the vacation can be interrupted at a service completion instant and the server begins a regular busy period with probability $1-\beta$ or continues the vacation with probability $\beta$.

The reneging times are assumed to be exponentially distributed. After the completion of service (which can be partial or incomplete), each customer may rejoin the system as a feedback customer for receiving another regular service with probability $v$ or he can leave the system with probability $\gamma$ where $v+\gamma=1$. A reneged customer can be retained in many cases by employing certain convincing mechanisms to stay in queue for completion of service. Thus, a reneged customer can be retained in the queuing system with some probability $\sigma$ or he may leave the queue without receiving service with probability $\delta(=1-\sigma)$.

We obtain the probability generating functions of the stationary state probabilities and deduce the explicit expressions of the system sizes when the server is in a normal service period and in a Bernoulli schedule vacation interruption, respectively. Various performance measures such as the mean system size, the proportion of customers served, the rate of abandonment due to impatience, and the mean sojourn time of a customer served are derived. Finally, we present some numerical examples to demonstrate how the various parameters of the model influence the behavior of the system.

The rest of the paper is arranged as follows: In Sect. 2, we describe the model, then we give in Sect. 3 the main result; the probability generating functions of the stationary state probabilities are obtained, then the explicit expressions of the system sizes when the server is in a normal service period and in a Bernoulli schedule vacation interruption are given, respectively. After that in Sect. 4, various performance measures such as the expected number of customers in the system when the server is on a working vacation period and in a normal busy period, the expected number of customers in the system, the proportion of customers served, the rate of abandonment due to impatience, and the mean sojourn time of a customer served are derived. Finally, we finish the paper by a small conclusion.

\section{Description of the model}

We consider the multiple working vacations $M / M / 1$ queueing system with Bernoulli schedule vacation interruptions, reneging, retention of reneged customers and feedback, where the customers become impatient due to the servers' vacation. Customers arrive according to a Poisson process with arrival rate $\lambda$. The service times during a normal service period, the service times during a working vacation period, and the vacation times are exponentially distributed with rates $\mu, \alpha$, and $\theta$, respectively.

The customers are assumed to be impatient during the multiple working vacations. Whenever a customer arrives at the system and finds the system is on working vacation, the customer activates an impatient timer $T$, which is exponentially distributed with rate $\vartheta$. If the server finishes the working vacation before the impatience timer expires, the customer remains in the system till his service completion.

However, if the impatience timer expires when the server is still on working vacation, the customer abandons the system, this time is reneging time of an individual customer. The reneged customer can be retained in the queue with some probability $\sigma$ or he may abandon the queue with complementary probability $\delta(1-\sigma)$. 
During the working vacation period, a customer is serviced at a lower rate, and, at the instants of the service completion, the vacation is interrupted and the server resumes a regular busy period with probability $1-\beta$ (if there are customers in the queue) or remains in the vacation with probability $\beta$. The inter-arrival times, service times, vacation duration times, and the impatient times all are taken to be mutually independent. The customers are served on a first come first-served queue discipline.

After completion of each service, the customer can either join the end of the queue with probability $v$ or he can leave the system with probability $\gamma$ where $\nu+\gamma=1$. The customers both newly arrived and those that are fed back are served in order in which they join the tail of original queue. We do not distinguish between the regular arrival and feedback arrival.

Let $N(t)$ denote the number of customers in the system at time $t$, and let $S(t)$ denote the state of the server at time $t$ with

$$
S(t)= \begin{cases}0, & \text { if the server is in working vacation period, } \\ 1, & \text { if the server is in normal busy period. }\end{cases}
$$

Then, the pair $\{(N(t), S t)) ; t \geq 0\}$ is a continuous-time Markov process with state space $\Omega=[\{(0,0) \cup$ $(i, j)\}, \quad i=1,2, \ldots, \quad j=0,1]$. Let $\pi_{i j}=\lim _{t \rightarrow \infty} \mathbb{P}\{N(t)=i, S(t)=j\}, \quad(i, j) \in \Omega$.

Let $\pi_{i 0}, i \geq 0$ be the probability that there are $i$ customers in the system when the server is in working vacation period and let $\pi_{i 1}, i \geq 1$ be the probability that there are $i$ customers in the system when the server is in normal busy period.

\section{Main result}

In this part of this paper, we study a stationary analysis for our model. At first, we develop the probability generating functions of the number of customers in the system when the server is in a working vacation period and in a normal service period, respectively. Then, we derive the explicit expressions for various performance measures.

Via the Markov process theory, we get the following set of steady-state equations:

$$
\begin{gathered}
\lambda \pi_{00}=(\delta \vartheta+\gamma \alpha) \pi_{10}+\gamma \mu \pi_{11} \\
(\lambda+\gamma \alpha+\theta+n \delta \vartheta) \pi_{n 0}=\lambda \pi_{n-10}+(\beta \gamma \alpha+(n+1) \delta \vartheta) \pi_{n+10}, \quad n \geq 1 \\
(\lambda+\gamma \mu) \pi_{11}=\theta \pi_{10}+\bar{\beta} \gamma \alpha \pi_{20}+\gamma \mu \pi_{21} \\
(\lambda+\gamma \mu) \pi_{n 1}=\theta \pi_{n 0}+\lambda \pi_{n-11}+\gamma \mu \pi_{n+11}+\bar{\beta} \gamma \alpha \pi_{n+10}, \quad n \geq 2
\end{gathered}
$$

where $\bar{\beta}=1-\beta$. Let us define the probability generating functions as

$$
\Pi_{0}(z)=\sum_{n=0} \pi_{n 0} z^{n}, \quad \Pi_{0}(z)=\sum_{n=1} \pi_{n 1} z^{n}
$$

where $\Pi_{0}(1)+\Pi_{1}(1)=1$ and $\Pi_{0}^{\prime}(z)=\sum_{n=1} n z^{n-1} \pi_{n 0}$.

Multiplying the appropriate power of $z^{n}$ in (1) and (2), in (3) and (4), respectively, then summing over all possible values of $n$ yield

$$
\begin{aligned}
& \delta \vartheta z(1-z) \Pi_{0}^{\prime}(z)+\left(\lambda z^{2}-(\lambda+\gamma \alpha+\theta) z+\beta \gamma \alpha\right) \Pi_{0}(z) \\
& \quad+\left(\gamma \mu \pi_{11}+(\theta+\bar{\beta} \gamma \alpha) \pi_{0,0}+\bar{\beta} \gamma \alpha \pi_{10}\right) z+\beta \gamma \alpha \pi_{0,0}(1-z)=0,
\end{aligned}
$$

and

$$
(\lambda z-\gamma \mu)(1-z) \Pi_{1}(z)=(\theta z+\bar{\beta} \gamma \alpha) \Pi_{0}(z)-\left(\gamma \mu \pi_{11}+(\theta+\bar{\beta} \gamma \alpha) \pi_{0,0}\right)+\bar{\beta} \gamma \alpha \pi_{10} z-\bar{\beta} \gamma \alpha(1-z) \pi_{0,0} .
$$


Then, solving Eq. (6), we get

$$
\Pi_{0}(z)=\frac{-\left(\gamma \mu \pi_{11}+(\theta+\bar{\beta} \gamma \alpha) \pi_{0,0}+\bar{\beta} \gamma \alpha \pi_{10}\right) \Phi_{1}(z)+\beta \gamma \alpha \Phi_{2}(z)}{\delta \vartheta e^{-(\lambda / \delta \vartheta) z} z^{\beta \gamma \alpha / \delta \vartheta}(1-z)^{(\theta+\bar{\beta} \gamma \alpha) / \delta \vartheta}},
$$

where

$$
\begin{aligned}
& \Phi_{1}(z)=\int_{0}^{z} \mathrm{e}^{-(\lambda / \delta \vartheta) x} x^{\beta \gamma \alpha / \delta \vartheta}(1-x)^{(\theta+\bar{\beta} \gamma \alpha) / \delta \vartheta-1} \mathrm{~d} x \\
& \Phi_{2}(z)=\int_{0}^{z} \mathrm{e}^{-(\lambda / \delta \vartheta) x} x^{\beta \gamma \alpha / \delta \vartheta-1}(1-x)^{(\theta+\bar{\beta} \gamma \alpha) / \delta \vartheta} \mathrm{d} x,
\end{aligned}
$$

where $\delta \neq 0, \quad \vartheta \neq 0, \quad x \neq 0$ and $\delta \vartheta \neq 1$.

Now, we should find the probabilities $\pi_{0,0}, \pi_{1,0}$, and $\pi_{1,1}$, then some important performance measures are obtained.

Assume that $\mathbb{E}\left(L_{0}\right)$ and $\mathbb{E}\left(L_{1}\right)$ are the expected number of customers in the system when the server is on a working vacation period and in a normal busy period, respectively.

So, adding (3) and (4) over all possible values of n, we obtain

$$
(\theta+\bar{\beta} \gamma \alpha) \Pi_{0}(1)=\left(\gamma \mu \pi_{11}+(\theta+\bar{\beta} \gamma \alpha) \pi_{0,0}\right)+\bar{\beta} \gamma \alpha \pi_{10}
$$

And using (7), we have

$$
\Pi_{1}(z)=\frac{(\theta z+\bar{\beta} \gamma \alpha) \Pi_{0}(z)-z(\theta+\bar{\beta} \gamma \alpha) \Pi_{0}(1)}{(\lambda z-\gamma \mu)(1-z)}-\frac{\bar{\beta} \gamma \alpha \pi_{0,0}}{(\lambda z-\gamma \mu)} .
$$

Using L'Hopital's rule, we get

$$
\Pi_{1}(1)=\left(\frac{\theta+\bar{\beta} \gamma \alpha}{\gamma \mu-\lambda}\right) \Pi_{0}^{\prime}(1)+\left(\frac{\bar{\beta} \gamma \alpha}{\gamma \mu-\lambda}\right)\left(\pi_{00}-\Pi_{0}(1)\right),
$$

where $\Pi_{0}^{\prime}(1)=\mathbb{E}\left(L_{0}\right)$; the expected number of customers in the system when the server is on a working vacation period.

Since $\Pi_{1}(1)=1-\Pi_{0}(1)$, and by using (13), we obtain

$$
\mathbb{E}\left(L_{0}\right)=\left(\frac{\gamma \mu-\lambda}{\theta+\bar{\beta} \gamma \alpha}\right)\left(1-\Pi_{0}(1)\right)-\left(\frac{\bar{\beta} \gamma \alpha}{\theta+\bar{\beta} \gamma \alpha}\right)\left(\pi_{00}-\Pi_{0}(1)\right) .
$$

Now, We have to deduce the proportion of time the server is on a working vacation period $\left(\Pi_{0}(1)\right)$, so that $\mathbb{E}\left(L_{0}\right)$ can be derived. Adding (2)-(4) and rearranging the terms, we get

$$
\begin{aligned}
\lambda \pi_{n 0}+\lambda \pi_{n 1}- & \left((\gamma \alpha+(n+1) \delta \vartheta) \pi_{n+1,0}+\gamma \mu \pi_{n+1,1}\right) \\
= & \lambda \pi_{n-1,0}+\lambda \pi_{n-1,1} \\
& +\left((\gamma \alpha+n \delta \vartheta) \pi_{n, 0}+\gamma \mu \pi_{n, 1}\right), \quad n \geq 1
\end{aligned}
$$

Using recursively (15), and applying (1)

$$
\lambda \pi_{n, 0}+\lambda \pi_{n, 1}=(\gamma \alpha+(n+1) \delta \vartheta) \pi_{n+1,0}+\gamma \mu \pi_{n+1,1}, \quad n \geq 0
$$

Adding over all possible values of $n$ in (16), we obtain

$$
\lambda \Pi_{0}(1)+\lambda \Pi_{1}(1)=\gamma \mu \Pi_{1}(1)+\gamma \alpha\left(\Pi_{0}(1)-\pi_{00}\right)+\delta \vartheta \sum_{n=0}^{\infty}(n+1) \pi_{n+1,0}, \quad n \geq 0 .
$$

Note that $\mathbb{E}\left(L_{0}\right)=\sum_{n=0}^{\infty}(n+1) \pi_{n+1,0}$ and $\Pi_{1}(1)=1-\Pi_{0}(1)$.

By substituting the value of $\mathbb{E}\left(L_{0}\right)$ from (14) in (17), we get

$$
\begin{aligned}
(\delta \vartheta+\theta+\bar{\beta} \gamma \alpha)(\gamma \mu-\lambda)= & (\delta \vartheta(\gamma \mu-\lambda)+(\gamma \mu-\gamma \alpha)(\theta+\bar{\beta} \gamma \alpha)-\delta \vartheta \bar{\beta} \gamma \alpha) \Pi_{0}(1) \\
& +(\delta \vartheta \bar{\beta} \gamma \alpha+\gamma \alpha(\theta+\bar{\beta} \gamma \alpha)) \pi_{0,0} .
\end{aligned}
$$


When $z \rightarrow \infty$ in (8) and using (6), (7) and (12), we get

$$
\Pi_{0}(1)=\frac{\mathrm{e}^{\lambda / \delta \vartheta}}{\delta \vartheta}\left(-(\theta+\bar{\beta} \gamma \alpha) \Pi_{0}(1) \Phi_{1}(1)+\bar{\beta} \gamma \alpha \pi_{00} \Phi_{2}(1)\right) \lim _{z \rightarrow+\infty}(1-z)^{-(\theta+\bar{\beta} \gamma \alpha) / \delta \vartheta}
$$

As, $0 \leq \Pi_{0}(1)=\sum_{n=0}^{\infty} \pi_{n, 0} \leq 1$ and $\lim _{z \rightarrow 1}(1-z)^{-(\theta+\gamma \bar{\beta} \alpha) / \delta \vartheta} \rightarrow \infty$, so we should have

$$
-(\theta+\bar{\beta} \gamma \alpha) \Pi_{0}(1) \Phi_{1}(1)+\beta \gamma \alpha \pi_{00} \Phi_{2}(1)=0 .
$$

Then, using (19) and (20), we get

$$
\pi_{0,0}=\frac{(\theta+\gamma \bar{\beta} \alpha) \Phi_{1}(1)}{\gamma \beta \alpha \Phi_{2}(1)} \Pi_{0}(1)
$$

and

$$
\begin{aligned}
\Pi_{0}(1)= & \left((\delta \vartheta+\theta+\gamma \bar{\beta} \alpha)(\gamma \mu-\lambda) \beta \Phi_{2}(1)\right) \times\left((\delta \bar{\beta} \vartheta+\theta+\gamma \bar{\beta} \alpha)(\theta+\gamma \bar{\beta} \alpha) \Phi_{1}(1)\right. \\
& \left.+(\delta \vartheta(\gamma \mu-\lambda)+\gamma(\mu-\alpha)(\theta+\gamma \bar{\beta} \alpha)-\delta \vartheta \bar{\beta} \gamma \alpha) \times \beta \Phi_{2}(1)\right)^{-1}
\end{aligned}
$$

Thus, $\mathbb{E}\left[L_{0}\right]$ is found from (14). Using (1) and (11) the unknowns $\pi_{1,0}$ and $\pi_{1,1}$ are obtained as follows:

$$
\begin{aligned}
& \pi_{1,0}=\frac{(\theta+\gamma \bar{\beta} \alpha)\left((\lambda+\theta+\gamma \bar{\beta} \alpha) \Phi_{1}(1)-\gamma \beta \alpha \Phi_{2}(1)\right)}{(\delta \vartheta+\gamma \beta \alpha) \gamma \beta \alpha \Phi_{2}(1)} \Pi_{0}(1), \\
\pi_{1,1}= & \left(\left((\theta+\gamma \bar{\beta} \alpha)\left\{(\delta \vartheta+\gamma \alpha) \beta \gamma \alpha \Phi_{2}(1)-\Phi_{1}(1)(\lambda \gamma \bar{\beta} \alpha+(\delta \vartheta+\gamma \alpha)(\theta+\gamma \bar{\beta} \alpha))\right\}\right)\right. \\
& \left.\times\left(\gamma \beta \mu \alpha \Phi_{2}(1)(\delta \vartheta+\gamma \beta \alpha)\right)^{-1}\right) \Pi_{0}(1) .
\end{aligned}
$$

Now, the stationary probabilities $\pi_{n, 0}$ and $\pi_{n, 1}$ can be derived by using (2)-(4) in terms of $\pi_{0,0}, \pi_{1,0}$, and $\pi_{1,1}$.

The expected number of customers in the system when the server is in a normal busy period $\mathbb{E}\left(L_{1}\right)$ can be obtained from (12).

By using L'Hopital's rule we get

$$
\begin{aligned}
\mathbb{E}\left(L_{1}\right)=\Pi_{1}^{\prime}(1)= & \frac{\theta+\gamma \bar{\beta} \alpha}{\gamma \mu-\lambda} \frac{\Pi_{0}^{\prime \prime}(1)}{2}+\frac{1}{(\theta+\gamma \bar{\beta} \alpha)(\gamma \mu-\lambda)} \\
& \times\left((\gamma \theta \mu+\lambda \gamma \bar{\beta} \alpha)\left(1-\Pi_{0}(1)\right)+\theta \gamma \bar{\beta} \alpha\left(\Pi_{0}(1)-\pi_{0,0}\right)\right) .
\end{aligned}
$$

Differentiating (6) twice at $z=1$, we obtain

$$
f^{\prime \prime}(1) \Pi_{0}(1)+2\left(f^{\prime}(1)-\delta \vartheta\right) \Pi_{0}^{\prime}(1)+(f(1)-2 \delta \vartheta) \Pi_{0}^{\prime \prime}(1)=0,
$$

where $f(1)=-(\theta+\gamma \bar{\beta} \alpha), f^{\prime}(1)=\lambda-(\gamma \alpha+\theta)$ and $f^{\prime \prime}(1)=2 \lambda$.

Then, from (26), we get

$$
\frac{1}{2} \Pi_{0}^{\prime \prime}(1)=\left(\frac{\lambda}{\theta+\gamma \bar{\beta} \alpha+2 \delta \vartheta}\right) \Pi_{0}(1)-\left(\frac{\delta \vartheta+\gamma \alpha+\theta-\lambda}{\theta+\gamma \bar{\beta} \alpha+2 \delta \vartheta}\right) \times \mathbb{E}\left(L_{0}\right)
$$

Using (27) and (14) in (25), we get $\mathbb{E}\left(L_{1}\right)$. The expected number of customers in the system can be computed as $\mathbb{E}(L)=\mathbb{E}\left(L_{0}\right)+\mathbb{E}\left(L_{1}\right)$. Now, we define the sojourn times, let $W$ be the total sojourn time of a customer in the system, evaluated from the instant of arrival till departure, with the departure either due to completion of service or as a consequence of abandonment. We have by Little's rule

$$
\mathbb{E}(W)=\frac{1}{\lambda}\left(\mathbb{E}\left(L_{0}\right)+\mathbb{E}\left(L_{1}\right)\right) .
$$


Table 1 Impact of $\vartheta$ and $\beta$ on some performance measures

\begin{tabular}{llllllllll}
\hline$\beta$ & $\vartheta$ & $\mathbb{E}\left(L_{0}\right)$ & $\mathbb{E}\left(L_{1}\right)$ & $\mathbb{E}(L)$ & $\mathbb{E}(W)$ & $\Delta$ & $\Theta$ & $\Gamma$ & $\Omega$ \\
\hline \multirow{3}{*}{0.15} & 0.4 & 0.38322 & 0.67317 & 1.05638 & 0.52819113 & 0.95018 & 0.09964 & 0.52912 & 0.47088 \\
& 1.2 & 0.36227 & 0.42851 & 0.79078 & 0.395392473 & 0.85871 & 0.28257 & 0.45684 & 0.54316 \\
& 2 & 0.33808 & 0.29520 & 0.63328 & 0.316639316 & 0.78025 & 0.43950 & 0.40317 & 0.59683 \\
0.5 & 0.4 & 0.44204 & 0.75376 & 1.19581 & 0.597903865 & 0.94253 & 0.11493 & 0.51473 & 0.48527 \\
& 1.2 & 0.40317 & 0.43885 & 0.84203 & 0.421012957 & 0.84276 & 0.31447 & 0.43569 & 0.56431 \\
& 2 & 0.36790 & 0.28641 & 0.65430 & 0.327150257 & 0.76087 & 0.47826 & 0.38126 & 0.61874 \\
0.9 & 0.4 & 0.55594 & 0.91678 & 1.47272 & 0.73636023 & 0.92773 & 0.14455 & 0.48943 & 0.51057 \\
& 1.2 & 0.46844 & 0.45451 & 0.92294 & 0.461472108 & 0.81731 & 0.36538 & 0.40222 & 0.59778 \\
& 2 & 0.41155 & 0.27219 & 0.68374 & 0.341872387 & 0.73249 & 0.53502 & 0.34925 & 0.65075 \\
1 & 0.4 & 0.60040 & 0.98064 & 1.58104 & 0.790519493 & 0.92195 & 0.15610 & 0.48032 & 0.51968 \\
& 1.2 & 0.48940 & 0.45930 & 0.94870 & 0.47434832 & 0.80913 & 0.38173 & 0.39152 & 0.60848 \\
& 2 & 0.42461 & 0.26764 & 0.69225 & 0.346124654 & 0.72400 & 0.55200 & 0.33969 & 0.66031 \\
\hline
\end{tabular}

Let $\Delta$ be the proportion of customers served, $\Theta$ be the rate of abandonment due to impatience. The expected number of customers served per unit of time is $\gamma \mu \Pi_{1}(1)-\gamma \alpha\left(\Pi_{0}(1)-\pi_{0,0}\right)$ signifying that the proportion of customers served is

$$
\Delta=\frac{1}{\lambda}\left(\gamma \mu \Pi_{1}(1)+\gamma \alpha\left(\Pi_{0}(1)-\pi_{0,0}\right)\right) .
$$

The rate of abandonment $\Theta$ of a customer due to impatience is given by

$$
\Theta=\delta \vartheta \mathbb{E}\left(L_{0}\right)=\lambda-\left(\gamma \mu \Pi_{1}(1)+\gamma \alpha\left(\Pi_{0}(1)-\pi_{0,0}\right)\right),
$$

which follows from (17). The probability that the system is in normal busy period $\Gamma$ and the probability that the system is in working vacation $\Omega$ are, respectively, given by

$$
\Gamma=\sum_{n=1}^{\infty} \pi_{n, 1}=\Pi_{1}(1), \quad \Omega=\sum_{n=0}^{\infty} \pi_{n, 0}=\Pi_{0}(1) .
$$

\section{Numerical results}

In this part of this paper, we present some numerical examples to demonstrate how the various parameters of the model influence the behavior of the system, and to show the impact of different parameters and its relationship with the expected number of customers when the system is on working vacation $\mathbb{E}\left(L_{0}\right)$, the expected number of customers when the system is on busy period $\mathbb{E}\left(L_{1}\right)$, the expected number of customers in the system $\mathbb{E}(L)$, the expected waiting time in the system $\mathbb{E}(W)$, the proportion of customers served $\Delta$, the rate of abandonment $\Theta$, the probability that the system is in normal busy period $\Gamma$ and the probability that the system is on working vacation $\Omega$.

$\checkmark$ Firstly, Let us present the evolution of the system by varying $\beta$, $\vartheta$, and $\alpha$.

- The parameters for Table 1 are taken as $\lambda=2, \mu=6, \theta=0.8, \alpha=3, \gamma=0.5$, and $\delta=0.65$.

Table 1 shows at first that for fixed $\vartheta, \mathbb{E}\left(L_{0}\right), \mathbb{E}\left(L_{1}\right), \mathbb{E}(L), \mathbb{E}(W), \Omega$ and $\Theta$ increase as $\beta$ increases, otherwise $\Delta$ and $\Gamma$ decease as $\beta$ increases. Now, for fixed $\beta, \mathbb{E}\left(L_{0}\right), \mathbb{E}\left(L_{1}\right), \mathbb{E}(L), \mathbb{E}(W), \Gamma$ and $\Delta$ decrease as $\vartheta$ increases. But the rate of abandonment $\Theta$ of a customer due to impatience and the probability that the system is in working vacation $\Omega$ increase as $\vartheta$ increases.

- The parameters for Table 2 are taken as $\lambda=3, \mu=7, \theta=0.4, \vartheta=0.6, \gamma=0.6$, and $\delta=0.7$.

Table 2 shows for fixed $\alpha, \mathbb{E}\left(L_{0}\right), \mathbb{E}(L) \mathbb{E}(W) \Omega$ and $\Theta$ increase as $\beta$ increases, otherwise $\mathbb{E}\left(L_{1}\right), \Delta$ and $\Gamma$ decrease.

However, for fixed $\beta, \mathbb{E}\left(L_{0}\right), \mathbb{E}\left(L_{1}\right), \mathbb{E}(L), \mathbb{E}(W), \Gamma$ and $\Theta$ decrease as $\alpha$ increases, otherwise, $\Delta$ and $\Omega$ increase as $\alpha$ increases. All these results (Tables 1,2 ) agree absolutely with our intuition.

$\checkmark$ Now, let us present the impact of service rate during vacation $\alpha$ on the expected number of customers in the system for different values of vacation rates $\theta$, while $\lambda=2 \mu=5, \vartheta=1, \beta=1, \gamma=0.6$ and $\delta=0.7$. The numerical results are given in Fig. 1.

From Fig. 1, we observe that the expected number of customers in the system decreases with the increase of $\alpha$, furthermore when $\alpha>1.5, \mathbb{E}(L)$ increases as $\theta$ increases, however, when $\alpha<1.5, \mathbb{E}(L)$ decreases when the vacation rate increases. 
Table 2 Impact of $\beta$ and $\alpha$ on some performance measures

\begin{tabular}{llllllllll}
\hline$\beta$ & $\alpha$ & $\mathbb{E}\left(L_{0}\right)$ & $\mathbb{E}\left(L_{1}\right)$ & $\mathbb{E}(L)$ & $\mathbb{E}(W)$ & $\Delta$ & $\Theta$ & $\Gamma$ & $\Omega$ \\
\hline \multirow{3}{*}{0.3} & 2 & 0.73780 & 2.14719 & 2.88498 & 0.961660758 & 0.89671 & 0.30987 & 0.55645 & 0.44355 \\
& 4 & 0.50582 & 1.85779 & 2.36360 & 0.787867677 & 0.92919 & 0.21244 & 0.51678 & 0.48322 \\
& 5 & 0.44269 & 1.74599 & 2.18868 & 0.72955893 & 0.93802 & 0.18593 & 0.49605 & 0.50395 \\
0.6 & 2 & 0.90276 & 2.11320 & 3.01595 & 1.00531821 & 0.87361 & 0.37916 & 0.52898 & 0.47102 \\
& 4 & 0.63494 & 1.77538 & 2.41032 & 0.803440036 & 0.91111 & 0.26667 & 0.47878 & 0.52122 \\
& 5 & 0.55563 & 1.64233 & 2.19796 & 0.732652234 & 0.92221 & 0.23336 & 0.45344 & 0.54656 \\
0.8 & 2 & 1.07762 & 2.07083 & 3.14845 & 1.049484825 & 0.84913 & 0.45260 & 0.50055 & 0.49945 \\
& 4 & 0.78531 & 1.67606 & 2.46137 & 0.820456865 & 0.89006 & 0.32983 & 0.43623 & 0.56377 \\
& 5 & 0.68812 & 1.51894 & 2.20706 & 0.735688075 & 0.90366 & 0.28901 & 0.40535 & 0.59465 \\
1 & 2 & 1.36460 & 1.99159 & 3.35620 & 1.118731794 & 0.80896 & 0.57313 & 0.45487 & 0.54513 \\
& 4 & 1.07313 & 1.47924 & 2.55237 & 0.85079094 & 0.84976 & 0.45072 & 0.35771 & 0.64229 \\
& 5 & 0.94565 & 1.27516 & 2.22081 & 0.740269278 & 0.86761 & 0.39717 & 0.31522 & 0.68478 \\
\hline
\end{tabular}
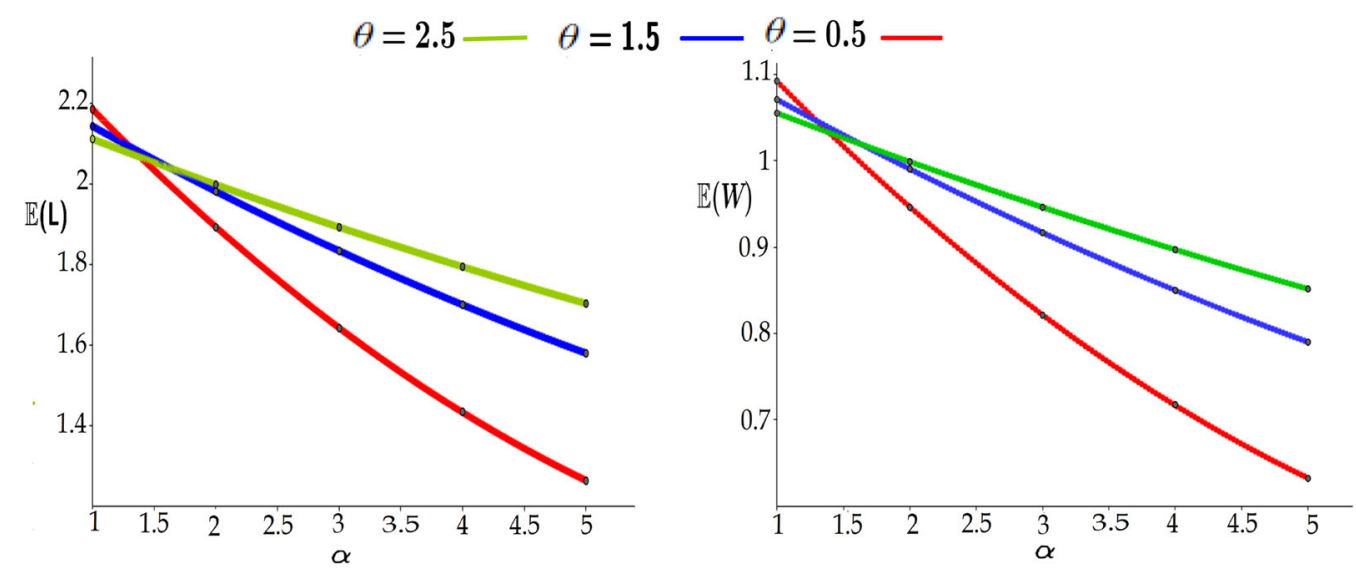

Fig. 1 Effect of $\alpha$ on $\mathbb{E}(L)$, effect of $\alpha$ on $\mathbb{E}(W)$

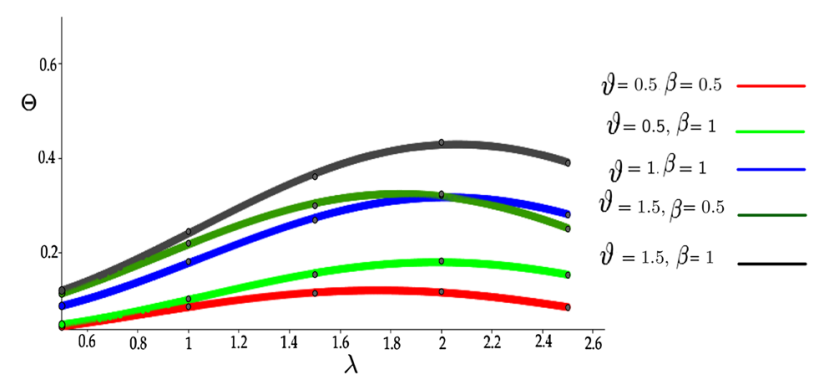

Fig. 2 Arrival rate $\lambda$ versus $\theta$

$\checkmark$ Next, we present the effect of arrival rate $\lambda$ on the rate of abandonment of a customer due to impatience, $\Theta$, and the effect of arrival rate $\lambda$ on $\mathbb{E}(L)$ and on $\mathbb{E}(W)$ for various parameters $\vartheta$ and $\beta$. We take $\mu=6$, $\theta=0.5, \alpha=4, \gamma=0.5$, and $\delta=0.5$.

Figure 2 shows that $\Theta$ first increases then diminishes with increasing of the arrival rate when $\beta=0.5$ and $\beta=1$, which agree absolutely with our expectation; the rate of abandonment of a customers increases when $\vartheta$ increases because more numbers of customers renege and leave the system.

Figure 3 shows the increases of $\mathbb{E}(L)$ and $\mathbb{E}(W)$ with the increases of $\lambda$. This result is absolutely reasonable.

$\checkmark$ Next, we present the dependence of the proportion of customers served and abandonment rate $\Delta$ and $\Theta$ with $\theta$ and $\vartheta$. Let $\lambda=3, \mu=5, \alpha=0.65, \beta=0.5, \delta=0.4$, and $\gamma=0.65$.

Figure 4 shows that for fixed $\theta, \Delta$ decreases and $\Theta$ increases when $\vartheta$ increases. Moreover, for fixed $\vartheta, \Delta$ increases and $\Theta$ decreases when $\theta$ increases.

$\checkmark$ Now, we present the dependence of the proportion of customers served and abandonment rate $\Delta$ and $\Theta$ to $\theta$ and $\delta$. Let $\lambda=3, \mu=5, \alpha=3, \beta=0.5, \vartheta=0.4$, and $\gamma=0.65$. 


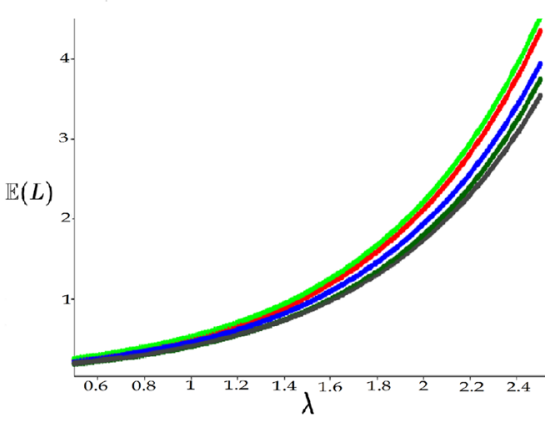

Fig. 3 Arrival rate $\lambda$ versus $\mathbb{E}(L)$, Arrival rate $\lambda$ versus $\mathbb{E}(W)$

$\Delta$

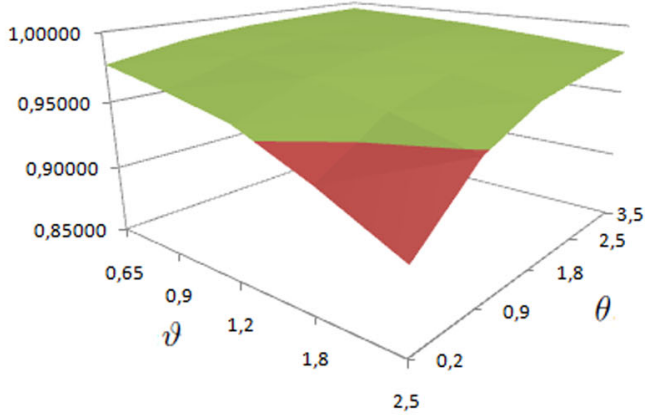

Fig. 4 Effect of $\theta$ and $\vartheta$ on $\Delta$ Effect of $\theta$ and $\vartheta$ on $\Theta$

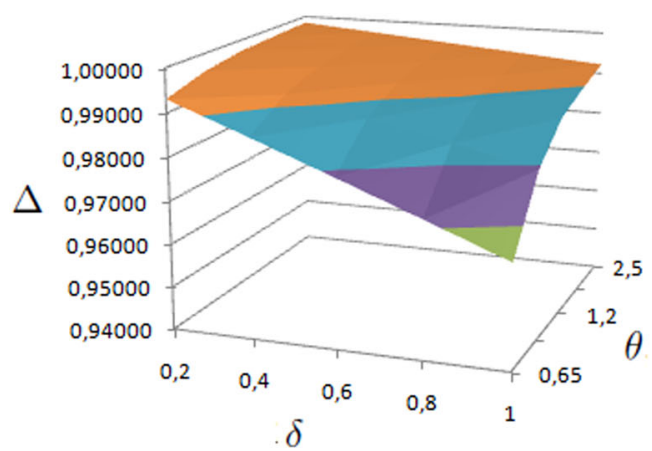

Fig. 5 Effect of $\theta$ and $\delta$ on $\Delta$, Effect of $\theta$ and $\delta$ on $\Theta$

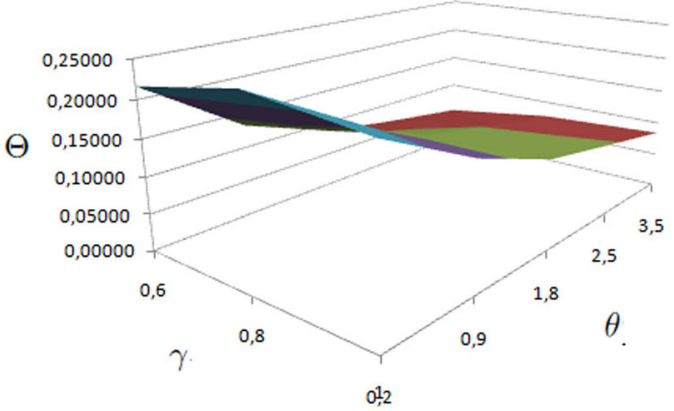

Fig. 6 Effect of $\theta$ and $\gamma$ on $\Theta$, effect of $\theta$ and $\gamma$ on $\Delta$
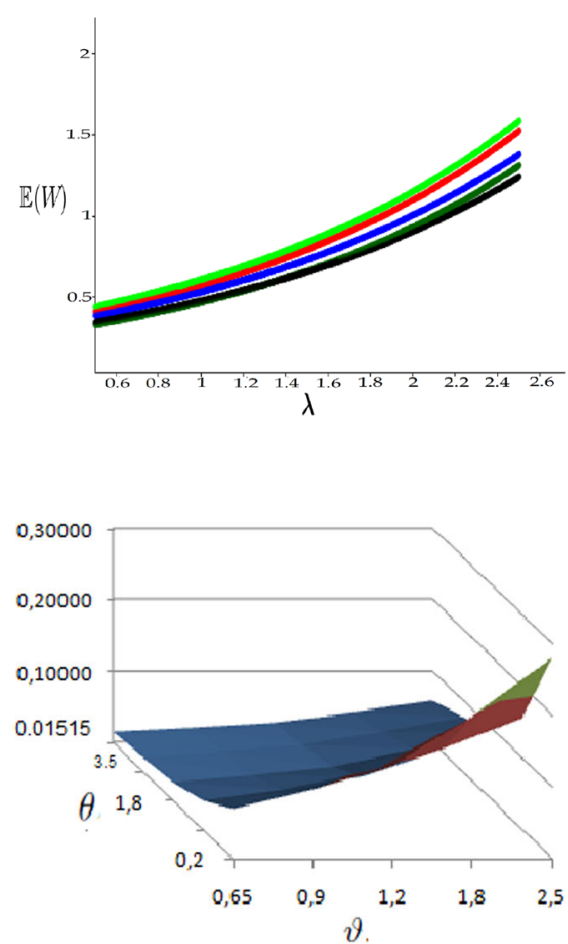

$\Theta$
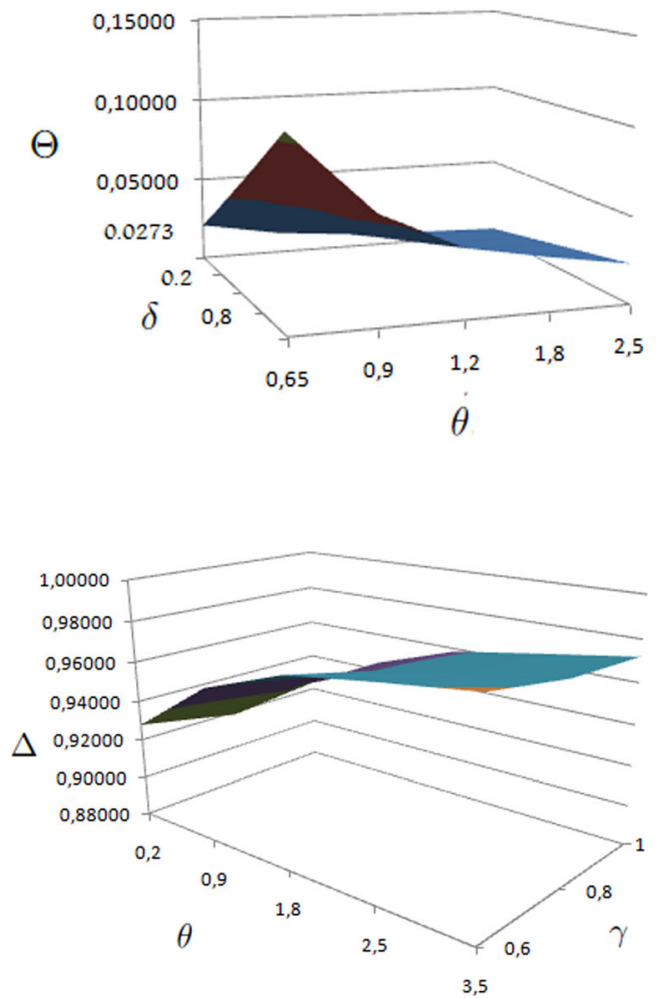

3,5 
Figure 5 shows that for fixed $\theta, \Delta$ decreases and $\Theta$ increases when $\delta$ increases. Moreover, for fixed $\delta, \Delta$ increases and $\Theta$ decreases when $\theta$ increases.

$\checkmark$ Finally, let's present dependence of the proportion of customers served $\Delta$ and abandonment rate $\Theta$ on $\theta$ and $\gamma$. We take $\lambda=3, \mu=7, \alpha=3, \beta=0.5, \vartheta=0.4$, and $\delta=0.65$.

Figure 6 shows that for fixed $\gamma, \Theta$ decreases and $\Delta$ increases when $\theta$ increases. Moreover, for fixed $\theta, \Theta$ increases and $\Delta$ decreases when $\gamma$ increases.

\section{Conclusion}

In this paper, an analysis of a feedback queueing system with reneging and retention of reneged customers, multiple working vacations and Bernoulli schedule vacation interruption is carried out, the explicit expressions for various performance measures are derived. Some numerical examples are presented to demonstrate how the various parameters of the model influence the behavior of the system. For further work, this model can be studied under the provision of time dependent arrival and service rate. The cost-profit analysis of the model can also be carried to study its economic analysis.

Open Access This article is distributed under the terms of the Creative Commons Attribution 4.0 International License (http:// creativecommons.org/licenses/by/4.0/), which permits unrestricted use, distribution, and reproduction in any medium, provided you give appropriate credit to the original author(s) and the source, provide a link to the Creative Commons license, and indicate if changes were made.

\section{References}

1. Abou El-Ata, M. O.; Hariri, A.M.A.: The $M / M / c / N$ queue with balking and reneging. Comput. Oper. Res. 19(13), 713-716 (1992)

2. Altman, E.; Yechiali, U.: Analysis of customers' impatience in queues with server vacations. Queueing Syst. Theory Appl. 52(4), 261-279 (2006)

3. Ancker Jr., C.J.; Gafarian, A.V.: Some queuing problems with balking and reneging: I. Oper. Res. 11(1), 88-100 (1963)

4. Ancker Jr., C.J.; Gafarian, A.V.: Some queuing problems with balking and reneging: II. Oper. Res. 11(6), $928-937$ (1963)

5. Ayyapan, G.; Muthu Ganapathi Subramanian, A.; Sekar, G.: $M / M / 1$ Retrial queueing system with loss and feedback under non-pre-emptive priority service by matrix Geometric method. Appl. Math. Sci. 4(48), 2379-2389 (2010)

6. Baba, Y.: The $M / P H / 1$ queue with working vacations and vacation interruption. J. Syst. Sci. Syst. Eng. 19(4), 496-503 (2010)

7. Chen, H.; Li, J.; Tian, N.: The $G I / M / I$ queue with phase type working vacations and vacation interruption. J. Appl. Math. Comput. 30(1-2), 121-141 (2009)

8. D’Avignon, G.R.; Disney, R.L.: Single server queue with state dependent feedback. INFOR 14, $71-85$ (1976)

9. El-Paoumy, M.S.; Nabwey, H.A.: The Poissonian queue with balking function, reneging and two heterogeneous servers. Int. J. Basic Appl. Sci. IJBAS-IJENS 11(6), 149-152 (2011)

10. Goswami, V.: Analysis of impatient customers in queues with Bernoulli schedule working vacations and vacation interruption. J. Stoch. 2014, 1-10, Article ID 207285 (2014)

11. Haight, F.A.: Queueing with balking. Biometrika 44, 360-369 (1957)

12. Haight, F.A.: Queueing with reneging. Metrika 2, 186-197 (1959)

13. Ke, J.C.; Wu, C.H.; Zhang, Z.G.: Recent developments in vacation queueing models: a short survey. Int. J. Oper. Res. 7(4), 3-8 (2010)

14. Kumar, R.; Sharma, S.K.: $M / M / 1 / N$ queuing system with retention of reneged customers. Pak. J. Stat. Oper. Res. 8, 859-866 (2012)

15. Kumar, R.; Sharma, S.K.: An $M / M / 1 / N$ queuing model with retention of reneged customers and balking. Am. J. Oper. Res. 2(1), 1-5 (2012)

16. Kumar, R.; Sharma, S.K.: A Markovian feedback queue with retention of reneged customers and balking. AMO-Adv. Model. Optim. 14(3), 681-688 (2012)

17. Laxmi, P.V.; Goswami, V.; Jyothsna, K.: Analysis of finite buffer Markovian queue with balking, reneging and working vacations. Int. J. Strateg. Decis. Sci. 4(1), 1-24 (2013)

18. Li, J.; Tian, N.: The $M / M / 1$ queue with working vacations and vacation interruptions. J. Syst. Sci. Syst. Eng. 16(1), 121-127 (2007)

19. Robert, E.: Reneging phenomenon of single channel queues. Math. Oper. Res. 4(2), 162-178 (1979)

20. Santhakumaran, A.; Thangaraj, V.A.: Single server queue with impatient and feedback customers. Inf. Manag. Sci. 11(3), 71-79 (2000)

21. Selvaraju, N.; Goswami, C.: Impatient customers in an $M / M / 1$ queue with single and multiple working vacations. Comput. Ind. Eng. 65(2), 207-215 (2013)

22. Sharma, S.K.; Kumar, R.: A Markovian feedback queue with retention of reneged customers and balking. AMO—Adv. Model. Optim. 14(3), 681-688 (2012) 
23. Takacs, L.: A single server queue with feedback. Bell Syst. Tech. J. 42, 134-149 (1963)

24. Thangaraj, V.; Vanitha, S.: On the analysis of $M / M / 1$ feedback queue with catastrophes using continued fractions. Int. J. Pure Appl. Math. 53(1), 131-151 (2009)

25. Tian, N.; Zhang, Z.G.: Vacation Queueing Models. Springer, New York (2006)

26. Wang, K.-H.; Chang, Y.-C.: Cost analysis of a finite $M / M / R$ queueing system with balking, reneging and server breakdowns. Math. Methods Oper. Res. 56(2), 169-180 (2002)

27. Yue, D.; Zhang, Y.; Yue, W.: Optimal performance analysis of an $M / M / 1 / N$ queue system with balking, reneging and server vacation. Int. J. Pure Appl. Math. 28(1), 101-115 (2006)

28. Yue, D.; Yue, W.; Xu, G.: Analysis of customers' impatience in an $M / M / 1$ queue with working vacations. J. Ind. Manag. Optim. 8(4), 895-908 (2012)

29. Zhang, M.; Hou, Z.: Performance analysis of $M / G / 1$ queue with working vacations and vacation interruption. J. Comput. Appl. Math. 234(10), 2977-2985 (2010)

30. Zhang, H.; Shi, D.: The $M / M / 1$ queue with Bernoulli schedule-controlled vacation and vacation interruption. Int. J. Inf. Manag. Sci. 20(4), 579-587 (2009) 